\title{
Impact of Spiritual Symptoms and Their Interactions on Health Services and Life Satisfaction
}

\author{
David A. Katerndabl, MD, MA \\ Department of Family \& Community Medi- \\ cine, University of Texas Health Science \\ Center at San Antonio, Texas
}

\begin{abstract}
PURPOSE Recent work suggests that the biopsychosocial model should be expanded to include the spiritual dimension as well. The purpose of this study was to assess the independent effects of spiritual symptoms and their interactions with biopsychosocial symptoms on health care utilization, extreme use of services, and life satisfaction among primary care patients.
\end{abstract}

METHODS Three hundred fifty-three adult waiting room patients at 2 primary care clinics completed the Biopsychosociospiritual Inventory (BioPSSI) as well as measures of life satisfaction and health care use. Hierarchical logistic regression analysis was performed with each outcome to determine whether adding spiritual symptoms and their interaction terms better accounted for outcomes than demographics, functional status, and chronic medical problems alone.

RESULTS Spiritual symptoms (alone or in interaction) were associated with 7 of the 10 outcomes and were particularly important to extreme use of health care services and life satisfaction. Among best-fit models, spiritual symptoms alone were significantly associated with any mental health use $(\beta=0.694, P \leq .05)$, fair-poor health status $(\beta=0.837, P \leq .05)$, and life lacking meaning $(\beta=1.214, P \leq .001)$.

CONCLUSIONS This study has shown the relevance of spiritual symptoms and their interactions to understanding health outcomes. Extreme utilization outcomes were related to the number of chronic problems, as well as to the social-spiritual interaction. Satisfaction outcomes were associated with physical and spiritual symptoms. These findings may have important implications for providing comprehensive, outcome-based care, as well as for modeling of research findings.

Ann Fam Med 2008;6:412-420. DOI: 10.1370/afm.886.

\section{INTRODUCTION}

S ince Engel ${ }^{1}$ described the biopsychosocial model and its clinical applications, ${ }^{2}$ this model has become a foundation of primary care. ${ }^{3,4}$ New models of care continue to emphasize the psychological and social aspects of illness in addition to the biomedical disorder. ${ }^{5-7}$ Biomedical, psychological, and social issues are important to everything from chronic pain ${ }^{8}$ and cerebral palsy ${ }^{9}$ to angina pectoris ${ }^{10}$ and inflammatory bowel syndrome, ${ }_{1}^{11}$ panic disorder, ${ }^{12}$ and depression. ${ }^{8,13,14}$ Yet, the biioopsychosocial model may be relevant to understanding a variety of clinical outcomes across disorders among primary care patients. ${ }^{15}$

Based on the consistent importance of religion and spirituality to health ${ }_{1}^{16-19}$ Onarecker and Sterling ${ }^{16}$ proposed that the biopsychosocial model be revised to include spirituality. This model is consistent with the definition of holism that "deals with health problems in their physical, psychological, social, cultural, and existential dimensions." ${ }^{20}$ In addition to a direct association between spirituality and clinical parameters, associations between biopsychosocial symptoms and spiritual factors ${ }^{21-25}$ suggest that 
spiritual symptoms may act synergistically with these other symptom dimensions to affect health. Even so, studies that have included spiritual assessments along with those of physical, psychological, and social symptoms have not sought such synergistic effects.

Does knowledge of spiritual symptoms contribute to our understanding of clinical outcomes above that which is explained by biopsychosocial symptoms? Does knowledge of interaction effects between spiritual symptoms and biopsychosocial symptoms add additional explanatory power? The purpose of this study was to assess the independent effects of spiritual symptoms and their interactions with biopsychosocial symptoms on health care utilization, extreme use of services, and life satisfaction among primary care patients.

\section{METHODS}

\section{Sample}

This study was conducted in 2 primary care clinics in San Antonio, Texas. The study was reviewed and approved by the Institutional Review Board for the University of Texas Health Science Center at San Antonio. English- and Spanish-speaking adult (aged 18 years and older) patients seeking nonacute care were approached in the waiting rooms and asked to participate. Those agreeing underwent informed consent and completed the questionnaire in a private setting.

\section{Instruments}

In addition to demographics, chronic medical problems, and medications, participants reported their levels of biopsychosociospiritual symptoms and functioning using the BIOPsychoSocioSpiritual Inventory (BioPSSI). ${ }^{26}$ Each symptom and activity item is rated from " 0 " (none of the time) to " 5 " (all of the time). This instrument consists of 5 scales (physical symptoms, psychological symptoms, social symptoms, spiritual symptoms, and impaired biopsychosociospiritual functioning) in which high scores indicate negative (or lack of positive) symptoms or limitations. All 5 scales have good concurrent validity (correlating with the number of scale-specific diagnoses, medications, and health care utilization, as well as scale-related satisfaction) and excellent internal consistency ( $\alpha=.862-.967)$. The 10 -item physical symptoms scale includes items concerning generalized nonspecific symptoms (ie, pain, weakness, dyspnea), whereas the 4 -item psychological symptoms scale includes items concerning anxiety and depression. The 8 -item social symptoms scale focuses on opportunities for social interaction and support, and the 7 -item spiritual symptom scale includes items concerning peace, harmony, and purpose (Table 1).
Although the 12-item impaired functional status scale assessed function in all 4 symptom dimensions, orthogonal (equamax) factor analysis showed that it was independent of the other scales. This scale, however, was still significantly correlated with the 4 symptom scales (Pearson $r=0.38-0.69$ ). The Spanish translation of the BioPSSI also showed excellent internal consistency for all 5 scales $(\alpha=.800-.965)$. In addition, Hispanic participants completing the English and Spanish versions did not differ significantly $(P>.3)$ when adjusted for age, sex, socioeconomic status, income, marital status, employment, and number of chronic medical problems.

Health care utilization was assessed using the Health Care Utilization Questionnaire. ${ }^{27}$ This instrument asked participants to report the number of visits during the previous 2 months to emergency settings, primary care and medical specialty settings, and mental health settings. Participants also reported the number of times during the previous year that they were admitted to a hospital. This instrument has good internal consistency.

Life satisfaction was assessed in 3 ways. Perceived health status was rated from "poor" to "excellent" on a 5 -point scale. ${ }^{28}$ Sense of meaning was assessed using a question from the FACIT-sp ${ }^{29}$ asking participants to rate how often during the previous month they felt that their life lacked meaning and purpose from " 0 " (none of the time) to "5" (all of the time). Finally, quality of life was assessed using a modified version of the Quality of Life Inventory (QOLI). ${ }^{30}$ For each area (ie, health, friends), participants were asked to rate the importance of the area to their happiness from " 0 " (not important) to "2" (extremely important) and to rate their satisfaction from " -3 " (very dissatisfied) to " +3 " (very satisfied). The QOLI was modified by additionally rating the importance of and satisfaction with their faith and spirituality using the same rating scales. The total quality-of-life score is computed by summing the importance-satisfaction products for each quality of life

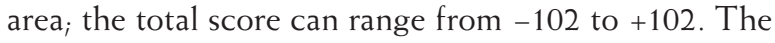
QOLI has good internal consistency and test-retest reliability, as well as concurrent and construct validity.

\section{Table 1. Items In The Spiritual Symptom Scale}

Item
1. Peaceful
2. A reason for living
3. Your life has been productive
4. Peace of mind
5. Sense of purpose
6. Able to reach down deep into yourself for comfort
7. Sense of harmony within yourself

1. Peaceful

2. A reason for living

3. Your life has been productive

Peace of mind

6. Able to reach down deep into yourself for comfort

7. Sense of harmony within yourself 


\section{Analysis}

Because the outcomes were not normally distributed, outcomes were dichotomized before analysis. Cut points were based on meaningfulness, published reports (definitions of extreme behavior), and rarity of the event. Any use of emergency settings, medical specialist and mental health settings, and hospitalization represent "any utilization" outcomes. Frequent use of primary care and ambulatory settings and polypharmacy represent "extreme utilization" outcomes. "Extreme utilization" was defined as that exhibited by the top $10 \%$ of participants; previous studies have traditionally used the top $5 \%$ as a cut point. ${ }^{31,32}$ "Frequent primary care use" was defined as more than 4 visits to a primary care physician in the previous 2 months. Other definitions have ranged from 15 visits in 3 years ${ }^{33}$ to 10 visits per year. ${ }^{34}$ "Frequent ambulatory use" (including medical specialists and mental health clinicians) was defined as more than 8 visits to any ambulatory health care worker in the previous 2 months. "Polypharmacy" was defined as more than 5 chronic medications. ${ }^{35}$ Finally, poor-fair health status, positive quality of life, and never lacking a sense of purpose represent "life satisfaction" outcomes.

Bivariate relationships between dichotomous outcome variables and scales were assessed using Student's t tests. To correct for multiple bivariate tests, Bonferroni's method was used. Thus, of the 50 bivariate analyses performed, only those with individual $P$ values of $\leq .001$ are significant at the experiment-wise $P \leq .05$ level when corrected for multiple comparisons. Hierarchical logistic regressions were performed to assess whether BioPSSI scales predict outcomes independent of their relation to demographic variables and the number of chronic medical problems. Adjusting for age, sex, socioeconomic status based on education and occupation, ${ }^{36}$ full-time employment, married status, and black race, each outcome was assessed with a series of 4 logistic regressions; income was not entered because of the high rate of missing data. In model 1 the number of chronic medical problems was added, whereas in model 2 the BioPSSI impaired functional status score was further added. In model 3 the 4 BioPSSI symptom scales were added to model 2. Finally, in model 4 , the 3 BioPSSI symptom scale interaction terms involving spiritual symptoms (physical $\times$ spiritual, psychological $\times$ spiritual, social $\times$ spiritual) were added. $P$ values $\leq .05$ were deemed significant, and $.05<P$ values $\leq .10$ were deemed as trending toward significance. Adjusted R ${ }^{2}$ was estimated using the Nagelkerke method, ${ }^{37}$ and goodness-of-fit was assessed using Hosmer-Lemeshow (HL) statistics. ${ }^{38}$ The model that showed an improvement in variance and a nonsignificant HL statistic was deemed the best fit. With an $\otimes$ level of .05 and seeking a moderate effect size, this regression analysis has a statistical power of $80 \%$.

\section{RESULTS}

This predominantly low-income, English-speaking, minority sample consisted of 237 women and 142 married individuals. Medically, 267 (74\%) reported at least 1 chronic medical problem and 211 (61\%) reported at least 1 chronic medication. The sample description is displayed in Table 2.

\section{Any Utilization}

Overall, 76 (26\%) participants reported at least 1 use of emergency services. Similarly, 77 (25\%) participants reported at least 1 visit to a specialist. Although 87 (26\%) reported at least 1 hospitalization, 49 (16\%) reported use of mental health services. Only mental health use showed significant differences for all 5 BioPSSI scales (Table 3). For emergency department use, model 2 with its association with impaired functional status was the optimal model (Table 4). Although better-fitting model 3 (HL $P=.358$ ) suggested that specialist use was related to physical

\section{Table 2. Sample Demographics $(n=353)$}

\begin{tabular}{|c|c|c|}
\hline Demographic & Number Missing & n (\%) \\
\hline Sex, female & 2 & 237 (67) \\
\hline Race/ethnicity & 3 & \\
\hline non-Hispanic white & & $108(31)$ \\
\hline Hispanic & & $207(59)$ \\
\hline Black & & $22(6)$ \\
\hline Other & & $13(4)$ \\
\hline Marital status & 3 & \\
\hline Married & & $142(40)$ \\
\hline Single & & $95(27)$ \\
\hline Other & & $113(32)$ \\
\hline Employment (full-time) & 5 & $88(25)$ \\
\hline Income & 59 & \\
\hline$<\$ 5,000$ & & $57(16)$ \\
\hline$\$ 5,000-\$ 19,999$ & & $118(33)$ \\
\hline$\$ 20,000-\$ 49,999$ & & $95(27)$ \\
\hline$\geq \$ 50,000$ & & $24(7)$ \\
\hline Social class & 27 & \\
\hline II & & $2(1)$ \\
\hline III & & $61(17)$ \\
\hline IV & & $72(20)$ \\
\hline V & & $191(54)$ \\
\hline Age, mean years \pm SD & 20 & $42.6 \pm 13.5$ \\
\hline $\begin{array}{l}\text { Chronic medical problems, } \\
\text { mean No. } \pm \text { SD }\end{array}$ & 0 & $2.1 \pm 2.0$ \\
\hline $\begin{array}{l}\text { Chronic medications, } \\
\text { mean No. } \pm \text { SD }\end{array}$ & 9 & $2.1 \pm 2.5$ \\
\hline
\end{tabular}


Table 3. Bivariate Biopsychosociospiritual Inventory Differences In Outcomes (Mean Scores)

\begin{tabular}{|c|c|c|c|c|c|}
\hline Outcomes, n & $\begin{array}{c}\text { Physical } \\
\text { Symptoms }\end{array}$ & $\begin{array}{l}\text { Psychological } \\
\text { Symptoms }\end{array}$ & $\begin{array}{c}\text { Social } \\
\text { Symptoms }\end{array}$ & $\begin{array}{c}\text { Spiritual } \\
\text { Symptoms }\end{array}$ & $\begin{array}{c}\text { Impaired } \\
\text { Functional Status }\end{array}$ \\
\hline \multicolumn{6}{|l|}{ Any utilization } \\
\hline \multicolumn{6}{|l|}{ Any emergency use } \\
\hline No, $n=221$ & $1.02(0.93)^{a, b}$ & $1.58(1.30)^{c}$ & $1.61(1.34)$ & $1.48(1.35)^{c}$ & $1.48(1.44)^{\mathrm{a}, \mathrm{b}}$ \\
\hline Yes, $n=76$ & $1.53(1.03)$ & $2.05(1.39)$ & $1.85(1.49)$ & $1.90(1.49)$ & $2.18(1.56)$ \\
\hline \multicolumn{6}{|l|}{ Any specialist use } \\
\hline No, $n=227$ & $0.99(0.90)^{a, b}$ & $1.56(1.27)^{d}$ & $1.71(1.36)$ & $1.51(1.37)^{c}$ & $1.46(1.42)^{a, b}$ \\
\hline Yes, $n=77$ & $1.74(1.14)$ & $2.18(1.49)$ & $1.69(1.51)$ & $1.94(1.52)$ & $2.40(1.65)$ \\
\hline \multicolumn{6}{|l|}{ Any mental health use } \\
\hline No, $n=265$ & $1.06(0.93)^{a, b}$ & $1.51(1.25)^{\mathrm{a}, \mathrm{b}}$ & $1.56(1.35)^{a, b}$ & $1.39(1.29)^{a, b}$ & $1.46(1.38)^{a, b}$ \\
\hline Yes, $n=49$ & $1.79(1.18)$ & $3.07(1.18)$ & $2.49(1.37)$ & $3.03(1.31)$ & $3.10(1.60)$ \\
\hline \multicolumn{6}{|l|}{ Any hospitalization } \\
\hline No, $n=241$ & $1.08(0.99)^{c}$ & $1.69(1.32)$ & $1.68(1.37)$ & $1.59(1.41)$ & $1.57(1.50)^{d}$ \\
\hline Yes, $n=87$ & $1.40(1.03)$ & $1.79(1.38)$ & $1.80(1.44)$ & $1.83(1.42)$ & $2.20(1.56)$ \\
\hline \multicolumn{6}{|l|}{ Extreme utilization } \\
\hline \multicolumn{6}{|l|}{ Frequent primary care use } \\
\hline$\leq 4$ Visits, $n=260$ & $1.01(0.91)^{\mathrm{a}, \mathrm{b}}$ & $1.63(1.29)^{a, b}$ & $1.66(1.36)$ & $1.55(1.40)^{\mathrm{e}}$ & $1.51(1.43)^{\mathrm{a}, \mathrm{b}}$ \\
\hline$>4$ Visits, $n=28$ & $2.10(1.07)$ & $2.58(1.65)$ & $2.03(1.51)$ & $2.29(1.41)$ & $3.04(1.53)$ \\
\hline \multicolumn{6}{|l|}{ Frequent ambulatory use } \\
\hline$\leq 8$ Visits, $\mathrm{n}=252$ & $1.02(0.91)^{a, b}$ & $1.58(1.29)^{\mathrm{a}, \mathrm{b}}$ & $1.64(1.37)$ & $1.50(1.36)^{d}$ & $1.45(1.36)^{a, b}$ \\
\hline$>8$ Visits, $\mathrm{n}=27$ & $1.86(1.05)$ & $2.70(1.46)$ & $2.00(1.51)$ & $2.32(1.54)$ & $3.35(1.67)$ \\
\hline \multicolumn{6}{|l|}{ Polypharmacy } \\
\hline$\leq 5$ Medications, $\mathrm{n}=307$ & $1.08(0.96)^{a, b}$ & $1.67(1.32)^{a, b}$ & $1.67(1.38)^{\mathrm{e}}$ & $1.61(1.43)^{\mathrm{e}}$ & $1.56(1.47)^{a, b}$ \\
\hline$>5$ Medications, $n=37$ & $2.17(1.12)$ & $2.75(1.31)$ & $2.31(1.24)$ & $2.29(1.41)$ & $3.09(1.46)$ \\
\hline \multicolumn{6}{|l|}{ Satisfaction } \\
\hline \multicolumn{6}{|l|}{ Fair-poor health status } \\
\hline No, $n=180$ & $0.74(0.72)^{a, b}$ & $1.16(1.08)^{\mathrm{a}, \mathrm{b}}$ & $1.29(1.24)^{a, b}$ & $1.05(1.11)^{a, b}$ & $1.04(1.21)^{\mathrm{a}, \mathrm{b}}$ \\
\hline Yes, $n=159$ & $1.80(1.08)$ & $2.43(1.30)$ & $2.23(1.39)$ & $2.39(1.41)$ & $2.61(1.47)$ \\
\hline \multicolumn{6}{|l|}{ Positive quality-of-life } \\
\hline No, $n=54$ & $1.92(1.06)^{a, b}$ & $3.03(1.22)^{a, b}$ & $2.55(1.38)^{a, b}$ & $3.10(1.22)^{a, b}$ & $3.21(1.45)^{\mathrm{a}, \mathrm{b}}$ \\
\hline Yes, $n=228$ & $1.01(0.89)$ & $1.40(1.18)$ & $1.52(1.29)$ & $1.29(1.23)$ & $1.38(1.34)$ \\
\hline \multicolumn{6}{|l|}{ Life lacking meaning } \\
\hline Never, $\mathrm{n}=149$ & $0.90(0.93)^{a, b}$ & $1.25(1.24)^{a, b}$ & $1.35(1.28)^{a, b}$ & $1.02(1.25)^{a, b}$ & $1.16(1.32)^{a, b}$ \\
\hline At least sometimes, $n=183$ & $1.39(1.02)$ & $2.17(1.28)$ & $2.04(1.40)$ & $2.24(1.34)$ & $2.22(1.55)$ \\
\hline \multicolumn{6}{|l|}{ Note: analyses determined by $t$ tests. } \\
\hline \multicolumn{6}{|l|}{$\begin{array}{l}\text { a } P \leq .001 \\
\text { b } P \leq .05 \text { by Bonferroni correction. } \\
\text { c } P \leq .05 . \\
\text { d } P \leq .005 . \\
\text { e } P \leq .01 .\end{array}$} \\
\hline
\end{tabular}

symptoms and lack of social symptoms, model 4 found associations with 2 interaction terms. For mental health use, although model 4 noted an association with psychological symptoms and was a better fit (HL $P=.610)$, it did not account for additional variance over model 3, which found associations with the number of chronic problems and spiritual (but not psychological) symptoms $(\alpha=0.694 ; P \leq .05)$. The lack of association between impaired functional status and any utilization in models 3 and 4 may reflect its correlation with symptom scores. Similarly, model 4 was not a significant improvement over model 3 for hospitalization and found that hospitalization was associated with both the number of chronic problems and impaired functional status as well as a lack of psychological symptoms.

\section{Extreme Utilization}

Although participants reported, on average, 1.65 primary care and 3.26 ambulatory visits, they also reported using 1.94 chronic medications. All bivariate comparisons were significant for impaired functional status as well as physical and psychological symptoms. Model 4 was an improvement over model 3 for all 3 extreme utilization outcomes (Table 5). Frequent 
primary care use was associated with the number of chronic problems, physical symptoms, and social-spiritual interaction. Similarly, frequent ambulatory use was related to the number of chronic problems and impaired functional status, as well as spiritual symptoms. Finally, polypharmacy was linked to the number of chronic problems and social symptoms, as well as social-spiritual interaction.
Table 4. Results of Regression Analyses ( $\beta$ ) for Health Services: Any Utilization

\begin{tabular}{|c|c|c|c|c|}
\hline Model & $\begin{array}{c}\text { Any } \\
\text { Emergency } \\
\text { Use }\end{array}$ & $\begin{array}{c}\text { Any } \\
\text { Specialist } \\
\text { Use }\end{array}$ & $\begin{array}{l}\text { Any Mental } \\
\text { Health Use }\end{array}$ & $\begin{array}{c}\text { Any } \\
\text { Hospitalization }\end{array}$ \\
\hline \multicolumn{5}{|l|}{ Model 1} \\
\hline $\begin{array}{l}\text { Number of } \\
\text { problems }\end{array}$ & $0.283^{a}$ & $0.271^{\mathrm{a}}$ & $0.563^{b}$ & $0.277^{c}$ \\
\hline$R^{2}(P)$ & $.100(.136)$ & $.158(.009)$ & $.268(.000)$ & $.122(.029)$ \\
\hline HL $P$ & .445 & .424 & .540 & .882 \\
\hline \multicolumn{5}{|l|}{ Model 2} \\
\hline $\begin{array}{l}\text { Number of } \\
\text { problems }\end{array}$ & 0.175 & 0.174 & $0.444^{c}$ & $0.218^{d}$ \\
\hline $\begin{array}{l}\text { Impaired functional } \\
\text { status }\end{array}$ & $0.372^{d}$ & $0.327^{d}$ & $0.520^{a}$ & 0.175 \\
\hline$R^{2}(P)$ & $.148(.035)$ & $.196(.003)$ & $.336(.000)$ & $.134(.029)$ \\
\hline HL $P$ & .526 & .442 & .786 & .275 \\
\hline \multicolumn{5}{|l|}{ Model 3} \\
\hline $\begin{array}{l}\text { Number of } \\
\text { problems }\end{array}$ & 0.172 & 0.126 & $0.498^{c}$ & $0.246^{d}$ \\
\hline $\begin{array}{l}\text { Impaired functional } \\
\text { status }\end{array}$ & 0.255 & 0.226 & 0.379 & $0.413^{d}$ \\
\hline \multicolumn{5}{|l|}{ Symptoms } \\
\hline Physical & 0.364 & $0.511^{\mathrm{e}}$ & -0.333 & 0.302 \\
\hline Psychological & -0.146 & -0.045 & 0.259 & $-0.804^{c}$ \\
\hline Social & 0.034 & $-0.364^{e}$ & -0.112 & -0.167 \\
\hline Spiritual & 0.102 & 0.032 & $0.694^{d}$ & 0.210 \\
\hline$R^{2}(P)$ & $.164(.101)$ & $.249(.002)$ & $.429(.000)$ & $.232(.002)$ \\
\hline HL $P$ & .918 & .358 & .510 & .428 \\
\hline \multicolumn{5}{|l|}{ Model 4} \\
\hline $\begin{array}{l}\text { Number of } \\
\text { problems }\end{array}$ & 0.198 & 0.116 & $0.508^{c}$ & $0.249^{d}$ \\
\hline $\begin{array}{l}\text { Impaired functional } \\
\text { status }\end{array}$ & 0.269 & 0.124 & 0.329 & $0.455^{d}$ \\
\hline \multicolumn{5}{|l|}{ Symptoms } \\
\hline Physical & 0.412 & 0.319 & 0.131 & 0.598 \\
\hline Psychological & -0.106 & $0.882^{d}$ & $1.310^{e}$ & $-1.051^{a}$ \\
\hline Social & 0.332 & $-1.082^{a}$ & -0.328 & 0.033 \\
\hline Spiritual & 0.586 & 0.269 & 0.744 & 0.359 \\
\hline \multicolumn{5}{|l|}{$\begin{array}{l}\text { Symptom } \\
\text { Interactions }\end{array}$} \\
\hline Physical-spiritual & 0.000 & 0.004 & 0.002 & -0.002 \\
\hline $\begin{array}{l}\text { Psychological- } \\
\text { spiritual }\end{array}$ & -0.002 & $-0.018^{d}$ & -0.006 & 0.005 \\
\hline Social-spiritual & -0.003 & $0.005^{e}$ & 0.001 & -0.002 \\
\hline$R^{2}(P)$ & $.183(.141)$ & $.314(.000)$ & $.434(.000)$ & $.252(.004)$ \\
\hline $\mathrm{HL} P$ & .350 & .004 & .610 & .536 \\
\hline $\begin{array}{l}\text { Note: Adjusted for age } \\
\text { HL } P=\text { Hosmer-Lemes } \\
\text { a } P \leq .01 . \\
\text { b } P \leq .001 . \\
\text { c } P \leq .005 . \\
\text { d } P \leq .05 . \\
\text { e } P \leq .1 .\end{array}$ & $\begin{array}{l}x \text {, socioeconomic s } \\
\text { N P value. }\end{array}$ & tus, race/ethnicity ( & ack), employment ( & l-time), married. \\
\hline
\end{tabular}

\section{Life Satisfaction}

Although 159 (47\%) subjects rated their health status as fair or poor, and $228(81 \%)$ rated their quality-of-life as positive, $149(45 \%)$ reported that their life always has meaning. All 5 BioPSSI scales showed significant bivariate differences for all 3 satisfaction outcomes. Model 3 showed that health status was related to physical and spiritual symptoms (Table 6). Model 4 also accounted for the most variance in a positive quality of life and found it was associated with fewer chronic problems, psychological but not physical symptoms, and the psychological-spiritual interaction (HL P =.690). The lack of association between impaired functional status and satisfaction in models 3 and 4 may reflect its correlation with symptom scores. Finally, model 4 was the best fit for lack of meaning (HLP $=.642)$ and found that it was related to impaired functional status, spiritual symptoms, and the social-spiritual interaction.

\section{DISCUSSION}

\section{Any Utilization}

Emergency department use was unrelated to any biospychosociospiritual symptoms, unlike previous studies that found emergency use was associated with psychological symptoms, stress, substance use, ${ }^{39-41}$ and parental religiosity $_{,}^{42}$ but also a lack of social support. ${ }^{32,39}$ Similarly, specialist use was possibly related to physical symptoms and a lack of social symptoms, but was only related to spiritual symptoms in model 4 , in which goodness of fit was poor. 


\begin{tabular}{|c|c|c|c|}
\hline Model & $\begin{array}{c}\text { Frequent } \\
\text { Primary } \\
\text { Care Use }\end{array}$ & $\begin{array}{c}\text { Frequent } \\
\text { Ambulatory } \\
\text { Use }\end{array}$ & $\begin{array}{c}\text { Poly- } \\
\text { pharmacy }\end{array}$ \\
\hline \multicolumn{4}{|l|}{ Model 1} \\
\hline Number of problems & $0.492^{\mathrm{a}}$ & $0.647^{b}$ & $0.810^{b}$ \\
\hline$R^{2}(P)$ & .199 (.109) & $.299(.002)$ & $.472(.000)$ \\
\hline $\mathrm{HL} P$ & .272 & .494 & .690 \\
\hline \multicolumn{4}{|l|}{ Model 2} \\
\hline Number of problems & $0.436^{c}$ & $0.561^{b}$ & $0.769^{b}$ \\
\hline Impaired functional status & 0.342 & $0.694^{d}$ & 0.394 \\
\hline$R^{2}(P)$ & $.227(.098)$ & $.394(.000)$ & $.496(.000)$ \\
\hline HL $P$ & .912 & .853 & .982 \\
\hline \multicolumn{4}{|l|}{ Model 3} \\
\hline Number of problems & $0.456^{c}$ & $0.580^{\mathrm{a}}$ & $0.833^{b}$ \\
\hline Impaired functional status & 0.083 & $0.709^{c}$ & 0.083 \\
\hline \multicolumn{4}{|l|}{ Symptoms } \\
\hline Physical & $1.370^{c}$ & 0.323 & 0.135 \\
\hline Psychological & -0.237 & -0.503 & 0.363 \\
\hline Social & -0.021 & -0.277 & 0.389 \\
\hline Spiritual & -0.477 & 0.378 & 0.003 \\
\hline$R^{2}(P)$ & $.327(.072)$ & $.419(.001)$ & $.546(.000)$ \\
\hline HL P & .784 & .717 & .994 \\
\hline \multicolumn{4}{|l|}{ Model 4} \\
\hline Number of problems & $0.457^{c}$ & $0.691^{\mathrm{a}}$ & $1.047^{b}$ \\
\hline Impaired functional status & 0.286 & $0.815^{c}$ & -0.007 \\
\hline \multicolumn{4}{|l|}{ Symptoms } \\
\hline Physical & $2.135^{\mathrm{e}}$ & -0.113 & -0.338 \\
\hline Psychological & 0.038 & 0.227 & 0.323 \\
\hline Social & 0.917 & 0.611 & $1.773^{c}$ \\
\hline Spiritual & 1.780 & $1.861^{\mathrm{e}}$ & 0.860 \\
\hline \multicolumn{4}{|l|}{ Symptom interactions } \\
\hline Physical-spiritual & -0.006 & 0.002 & 0.003 \\
\hline Psychological-spiritual & -0.008 & -0.016 & -0.001 \\
\hline Social-spiritual & $-0.010^{e}$ & -0.007 & $-0.009 c$ \\
\hline$R^{2}(P)$ & $.413(.045)$ & $.475(.001)$ & $.594(.000)$ \\
\hline HL $P$ & .951 & .954 & .817 \\
\hline
\end{tabular}

Note: Adjusted for age, sex, socioeconomic status, race/ethnicity (black), employment (fulltime), married.

HL $P=$ Hosmer-Lemeshow $P$ value.

a $P \leq .005$

${ }^{b} P \leq .001$.

c $P \leq .05$.

d $P \leq .01$.

e $P \leq 1$.

Although mental health use was associated with psychological symptoms in model 4 , it was not linked to psychological symptoms in model 3. Model 3 found that use was associated with the number of chronic problems and spiritual symptoms instead. This finding agrees with the association between mental health care and infrequent church attendance reported by Schiller and Levin. ${ }^{42}$ The association with spiritual symptoms instead of psychological symptoms in this study is unexpected. The change in correlates as we transition from model 3 to model 4 suggests a strong interdependency between psychological and spiritual symptoms. ${ }^{43}$ Only when the spiritual interaction terms were added did a significant relationship with psychological symptoms manifest itself.

Finally, though previous work found an association between religious denomination and both hospitalization and length of hospital stay ${ }_{1}^{42,44}$ as well as associations between religious/spiritual variables with acute hospitalizations and long-term care in elders, ${ }^{45}$ no relationship between hospitalization and spiritual symptoms or their interactions was found in this study.

\section{Extreme Utilization}

For all 3 extreme utilization variables, spiritual symptoms were significant. Frequent primary care use was associated with the number of chronic problems, possibly physical symptoms, and the social-spiritual interaction. Previous research linked frequent primary care office to the number of chronic problems ${ }^{46}$ physical symptoms ${ }^{33,40}$ psychological symptoms, ${ }^{31,33,40,46}$ poor social support, ${ }^{46}$ and religious denomination. ${ }^{42}$ King and Pearson ${ }^{47}$ found that patients who attended religious services were more likely to report continuity with a regular health care clinician.

Frequent ambulatory care use was linked to the number of chronic problems and possibly spiritual symptoms but was also related to impaired functional status. Previous research has linked ambulatory utilization with medical burden, ${ }^{48}$ impaired function, ${ }^{48}$ physical symptoms ${ }^{49}$ psychological symptoms ${ }_{1}^{49,50}$ social problems, ${ }^{49}$ and religiosity. ${ }^{42}$

Finally, polypharmacy was related to the number of chronic problems and social symptoms, as well as the social-spiritual interaction. Although prior studies linked polypharmacy to general symptomatology, ${ }^{50}$ no distinction between types of symptoms was made. The strong relationship between polypharmacy and social symptoms is unexpected, suggesting that a lack of social relationships and support may be an important factor is general symptomatology and use of medications.

\section{Life Satisfaction}

The satisfaction outcomes were strongly related to spiritual symptoms. Fair-poor health status was associated 
Table 6. Results of Regression Analyses ( $\beta$ ) for Health Services: Satisfaction

\begin{tabular}{|c|c|c|c|}
\hline Model & $\begin{array}{l}\text { Fair-Poor } \\
\text { Health Status }\end{array}$ & $\begin{array}{l}\text { Positive } \\
\text { Quality of Life }\end{array}$ & $\begin{array}{l}\text { Life Lacking } \\
\text { Meaning }\end{array}$ \\
\hline \multicolumn{4}{|l|}{ Model 1} \\
\hline Number of problems & $0.422^{\mathrm{a}}$ & $-0.676^{a}$ & $0.194^{b}$ \\
\hline$R^{2}(P)$ & $.214(0.000)$ & $.479(0.000)$ & $.103(0.046)$ \\
\hline $\mathrm{HL} P$ & 0.180 & 0.712 & 0.929 \\
\hline \multicolumn{4}{|l|}{ Model 2} \\
\hline Number of problems & $0.215^{c}$ & $-0.540^{a}$ & -0.017 \\
\hline Impaired functional status & $0.610^{\mathrm{a}}$ & $-0.601^{d}$ & $0.618^{\mathrm{a}}$ \\
\hline$R^{2}(P)$ & $.325(.000)$ & $.545(.000)$ & $.231(.000)$ \\
\hline $\mathrm{HL} P$ & .360 & .933 & .982 \\
\hline \multicolumn{4}{|l|}{ Model 3} \\
\hline Number of problems & 0.201 & $-0.717^{d}$ & 0.005 \\
\hline Impaired functional status & 0.061 & 0.115 & $0.445^{b}$ \\
\hline \multicolumn{4}{|l|}{ Symptoms } \\
\hline Physical & $1.149^{\mathrm{a}}$ & $-1.047^{b}$ & -0.059 \\
\hline Psychological & -0.144 & 0.140 & -0.051 \\
\hline Social & 0.079 & 0.096 & 0.003 \\
\hline Spiritual & $0.654^{d}$ & $-1.373^{d}$ & $0.567^{e}$ \\
\hline$R^{2}(P)$ & $.503(.000)$ & $.725(.000)$ & $.306(.000)$ \\
\hline HL P & .722 & .734 & .263 \\
\hline \multicolumn{4}{|l|}{ Model 4} \\
\hline Number of problems & 0.209 & $-0.766^{d}$ & 0.041 \\
\hline Impaired functional status & 0.085 & -0.012 & $0.464^{b}$ \\
\hline \multicolumn{4}{|l|}{ Symptoms } \\
\hline Physical & $1.552^{\mathrm{a}}$ & -1.849 & 0.277 \\
\hline Psychological & -0.149 & $1.902^{\mathrm{b}}$ & -0.189 \\
\hline Social & 0.075 & -1.003 & 0.407 \\
\hline Spiritual & $0.837^{b}$ & -1.090 & $1.214^{\mathrm{a}}$ \\
\hline \multicolumn{4}{|l|}{ Symptom interactions } \\
\hline Physical-spiritual & -0.003 & 0.006 & -0.003 \\
\hline Psychological-spiritual & 0.001 & $-0.027^{b}$ & 0.002 \\
\hline Social-spiritual & 0.000 & 0.006 & $-0.004^{b}$ \\
\hline$R^{2}(P)$ & $.511(.000)$ & $.759(.000)$ & $.345(.000)$ \\
\hline $\mathrm{HL} P$ & .725 & .690 & .642 \\
\hline \multicolumn{4}{|c|}{$\begin{array}{l}\text { Note: Adjusted for age, sex, socioeconomic status, race/ethnicity (black), employment (full-time), } \\
\text { married. }\end{array}$} \\
\hline \multicolumn{4}{|c|}{ HL $P=$ Hosmer-Lemeshow $P$ value. } \\
\hline $\begin{array}{l}\text { a } P \leq .001 . \\
\text { b } P \leq .05 \\
\text { c } P \leq . .1 \\
\text { d } P \leq .005 \\
\text { e } P \leq .01\end{array}$ & & & \\
\hline
\end{tabular}

quality of life to spirituality, ${ }_{1}^{55}$ not all studies agree. ${ }^{56}$ The unexpected pattern seen here may reflect a complex relation between psychological and spiritual symptoms, so that model 3 shows an inverse relationship with spiritual symptoms alone but model 4 shows that psychological symptoms are positively associated with positive quality of life whereas their interaction term is inversely related. More research in this area is needed.

Finally, a lack of meaning was associated with impaired functional status, spiritual symptoms, and a social-spiritual interaction. A sense of purpose in life is associated with better quality of life ${ }^{57}$ and perceived health, ${ }^{57}$ fewer psychological symptoms, ${ }^{58}$ and better abstinence rates. ${ }^{59}$ Meaning in life is associated with religiosity ${ }^{60}$ and social desirability, ${ }_{1}^{61}$ consistent with the importance of spirituality and socialspiritual interaction.

\section{Implications}

Although prior research has not included spiritual symptoms or their interaction effects in most cases, most of their results agree with those of this study. This study shows the importance of spiritual symptoms and interactions, sometimes more than biopsychosocial symptoms, in understanding health outcomes from a research perspective, confirming a complex relationship. ${ }^{62}$ This finding implies that we should expect nonlinear relationships to exist and unpredictability in these outcomes to be the rule rather than the exception. We must consider nonlinear approaches to modeling them and should address the full array of symptoms and potential treatment approaches when providing care. ${ }^{63}$

with physical and spiritual symptoms. Previous studies found associations between poor health status and disability, and psychological symptoms, ${ }^{51}$ infrequent church attendance, ${ }^{52}$ and spirituality. ${ }^{53}$

A positive quality of life was related to fewer chronic problems, psychological but lack of physical symptoms, and a psychological-spiritual interaction. Previous research linked quality of life with physical symptoms, psychological symptoms, and social and family symptoms. ${ }^{54}$ Although most studies linked
Clinically, this study has implications. First, it suggests that, when attempting to understand high utilization rates in patients with poor quality of life, clinicians should inquire about spiritual issues. Second, whereas positive interaction coefficients may suggest synergy between symptom dimensions, negative coefficients may suggest complementary effects; in fact, all significant interaction terms in best-fitting model 4 outcomes are indeed negative interaction effects. Thus, positive interactions suggest that symptoms in 
one dimension can be mitigated by promoting health in another dimension. Negative interactions may imply that the adverse effects of symptoms in one dimension complement symptoms in the other dimension. Lack of social interaction, for example, may promote spiritual well-being by facilitating introspection. Third, when patients respond in unexpected ways, clinicians should assess the possible role of spiritual problems.

\section{Limitations}

This study has several limitations. The predominance of lower income, Hispanic participants raises questions about its generalizability to other populations. Subpopulations within the Hispanic community may differ even though previous studies have suggested that Hispanic populations within the southwestern United States are similar among themselves. ${ }^{64}$ This study was conducted in a clinic population and may not apply to the general population. Selection bias may be present. Some outcomes were based on a single question and are subject to instability. Although the cut points used to define extremes in health services are similar to those of previous studies, there are no agreed-upon definitions for excessive utilization. Current problems and medications as well as 2-month utilization are based on selfreport and patient recall. Even so, an earlier study suggests that even depressed patients can accurately recall their utilization for the previous 2 months. ${ }^{65}$ Finally, the demographic covariates controlled for in the models may be incomplete. Low income is linked to decreased social support, as well as increased chronic medical problems and psychological symptoms. ${ }^{66}$

In conclusion, that spiritual symptoms (alone or in interaction) were associated with 7 of the 10 outcomes has important implications for providing comprehensive, outcome-based care as well as for modeling of research findings.

To read or post commentaries in response to this article, see it online at http://www.annfammed.org/cgi/content/full/6/5/412.

Submitted January 25, 2008; submitted, revised, May 30, 2008; accepted June 24, 2008.

Key words: Quality of life; health status; spirituality; health care delivery; mental health; delivery of health care

Funding support: This study was supported by the Texas Academy of Family Physicians Foundation.

\section{References}

1. Engel GL. The need for a new medical model: a challenge for biomedicine. Science. 1977;196(4286):129-136.

2. Engel GL. Clinical application of the biopsychosocial model. Am J Psychiatry. 1980;137(5):535-544.

3. Brody $\mathrm{H}$. The validation of the biopsychosocial model. J Fam Pract. 1990; 30(3):271-272
4. Masters KS. Recurrent abdominal pain, medical intervention, and biofeedback. Appl Psychophysiol Biofeedback. 2006;31(2):155-165.

5. Andersen RM. Revisiting the behavioral model and access to medical care. J Health Soc Behav. 1995;36(1):1-10.

6. Evans RG, Stoddart GL. Producing health, consuming health care. Soc Sci Med. 1990;31(12):1347-1363.

7. Gelberg L, Andersen RM, Leake BD. Behavioral Model for vulnerable populations. Health Serv Res. 2000;34(6):1273-1302.

8. Campbell L, Clauw D, Daniel J, Keefe FJ. Persistent pain and depression. Biol Psychiatry. 2003;54(3):399-409.

9. Cox BJ. Predictors of depression in adults with cerebral palsy. Dissertation Abstracts International section B 1999; 60(4-B): 1847.

10. Medalie JH. Angina pectoris: A validation of biopsychosocial model. J Fam Pract. 1990;30(3):273-280.

11. Halpert A, Drossman D. Biopsychosocial issues in irritable bowel syndrome. J Clin Gastroenterol. 2005;39(8):665-669.

12. Noyes $R$ Jr. Is panioc disorder a disease for the medical model? Psychosomatics. 1987;28(11):582-586.

13. Myers HF. Biopsychosocial perspective on depression in AfricanAmericans. In: Lin KM, Poland RE, et al, eds. Psychopharmacology And Psychobiology Of Ethnicity. Progress In Psychiatry Series, No. 39. Washington, DC: American Psychiatric Association; 1993.

14. Kaslow NJ, Baskin ML, Wyckoff SC. Biopsychosocial treatment approach for depressed children and adolescents. In: Kaslow FW, ed. Comprehensive Handbook Of Psychotherapy: Integrative/Eclectic. Vol 4. New York, NY: John Wiley \& Sons, Inc; 2002.

15. De Jonge $P$, Huyse FJ, Joris PJS, Sollner W, Stiefel FC. Operationalization of biopsychosocial case complexity in general health care. Aust N ZJ Psychiatry. 2005;39(9):795-799.

16. Onarecker CD, Sterling BC. Addressing your patients' spiritual needs. Fam Pract Manag. 1995:44-49.

17. Matthews DA, Larson DB, Barry CP. The Faith Factor (vol 1). Washington, DC: National Institute for Healthcare Research, 1993.

18. Larson DB. The Faith Factor. Vol 2. Washington, DC: National Institute for Healthcare Research; 1993.

19. Matthews DA, Larson DB. The Faith Factor (vol 3). Washington, DC: National Institute for Healthcare Research, 1995.

20. Freeman J. Toward a definition of holism. Br J Gen Pract. 2005;55(511):154-155.

21. Gartner J, Allen GD, Larson DB. Religious commitment and mental health - a review of the empirical literature. J Psychol Theol. 1991;19(1):6-25.

22. Highfield MF. Spiritual health of oncology patients. Nurse and patient perspectives. Cancer Nurs. 1992;15(1):1-8.

23. Hannay DR. Religion and health. Soc Sci Med. 1980;14A:683-685.

24. Bergin AE. Religiosity and mental health. Prof Psychol Res Pr. 1983;14:170-184.

25. Ellison CG, George LK. Religious involvement, social ties and social support in a southeastern community. J Sci Study Relig. 1994;33(1):46-61.

26. Katerndahl D, Oyiriaru D. Assessing the Biopsychosociospiritual Model in primary care: development of the BioPsychoSocioSpiritual Inventory (BioPSSI). Int J Psychiatry Med. 2007;37(4):393-414.

27. Katerndahl DA, Realini JP. Use of health care services by persons with panic symptoms. Psychiatr Serv. 1997;48(8):1027-1032.

28. Ware JE Jr, Sherbourne CD. The MOS 36-item short form health survey (SF-36), I. Conceptual framework and item selection. Med Care. 1992;30(6):473-483.

29. Cella D. Functional Assessment of Chronic Illness Therapy Manual. Version 4. Chicago, IL: Center on Outcomes Research and Education; 1997. 
30. Frisch MB. Quality of Life Inventory (QOLI). Minneapolis, MN: National Computer Systems; 1994.

31. Ford JD, Trestman RL, Steinberg K, Tennen H, Allen S. Prospective association of anxiety, depressive and addictive disorders with high utilization of primary, specialty, and emergency medical care. Soc Sci Med. 2004;58(11):2145-2148

32. Pasic J, Russo J, Roy-Byrne P. High utilizers of psychiatric emergency services. Psychiatr Serv. 2005;56(6):678-684.

33. Glaser JP, van Os J, Portegiijs PJM, Myin-Germeys I. Childhood trauma and emotional reactivity to daily life stress in adult frequent attenders of general practitioners. J Psychosom Res. 2006;61(2):229-236

34. Naessens JM, Baird MA, Van Houten HK, Vanness DJ, Campbell CR. Predicting persistently high primary care use. Ann Fam Med. 2005;3(4):324-330

35. Steinman MA, Landefeld CS, Rosenthal GE, Berthenthal D, Sen S, Kaboli PJ. Polypharmacy and prescribing quality in older people. J Am Geriatr Soc. 2006;54(10):1516-1523.

36. Hollingshead A, Redlich F. Social Class and Mental Illness. New York, NY: John Wiley; 1958..

37. Nagelkerke NJD. A note on a general definition of the coefficient of determination. Biometrika. 1991;78(3):691-692.

38. Hosmer DW, Lemeshow S. Applied Logistic Regression. 2nd ed. New York, NY: John Wiley and Sons; 2000.

39. O'Brien GM, Stein MD, Zierlers S, Shapiro M, O'Sullivan P, Woolard $\mathrm{R}$. Use of the ED as a regular source of care. Ann Emerg Med. 1997;30(3):286-291.

40. Neighbors CJ, Zywiak WH, Stout RL, Hoffmann NG. Psychobehavioral risk factors, substance treatment engagement and clinical outcomes as predictors of emergency department use and medical hospitalization. J Stud Alcohol. 2005;66(2):295-304.

41. Wilson KM, Klein JD. Adolescents who use the emergency department as their usual source of care. Arch Pediatr Adolesc Med. 2000;154(4):361-365.

42. Schiller PL, Levin JS. Is there a religious factor in health care utilization? Soc Sci Med. 1988;27(12):1369-1379.

43. Daaleman TP, Kaufman JS. Spirituality and depressive symptoms in primary care outpatients. South Med J. 2006;99(12):1340-1344.

44. Koenig HG, Larson DB. Use of hospital services, religious atten dance, and religious affiliation. South Med J. 1998;91(10):925-932.

45. Koenig HG, George LK, Titus P, Meador KG. Religion, spirituality, and acute care hospitalization and long-term care by older patients. Arch Intern Med. 2004;164(14):1579-1585.

46. Daugird AJ, Spencer DC. Characteristics of patients who highly utilize telephone medical care in aprivate practice. J Fam Pract. 1989;29(1):59-63.

47. King DE, Pearson WS. Religious attendance and continuity of care. Int J Psychiatry Med. 2003;33(4):377-389.

48. Kravitz RL, Greenfield S, Rogers W, et al. Difference in the mix of patients among medical specialties and systems of care. JAMA. 1992;267(12):1617-1623.
49. Uphold CR, Mkanta WN. Use of health care services among persons living with HIV infection. AIDS Patient Care Standards. 2005;19:473-485.

50. Goodwin R, Andersen RM. Use of the Behavioral Model of Health Care Use to identify correlates of use of treatment for panic attacks in the community. Soc Psychiatry Psychiatr Epidemiol. 2002;37(5):212-219.

51. Badger TA, McNiece C, Gagan MJ. Depression, service need, and use in vulnerable populations. Arch Psychiatr Nurs. 2000;14(4):173-182.

52. Levin JS, Vanderpool HY. Is frequent religious attendance really conducive to better health? Soc Sci Med. 1987;24(7):589-600.

53. Daaleman TP, Perera S, Studenski SA. Religion, spirituality, and health status in geriatric outpatients. Ann Fam Med. 2004;2(1):49-53.

54. Heller RF, Lim L, Valenti L, Knapp J. Predictors of quality of life after hospital admission for heart attack or angina. Int J Cardiol. 1997;59(2):161-166.

55. Brady MJ, Peterman AH, Fitchett G, Mo M, Cella D. Case for including spirituality in quality of life measurement in oncology. Psychooncology. 1999;8(5):417-428.

56. Metzger LK. Religion/spirituality and health outcomes in a secondary analysis of data on patients recovering from an acute myocardial infarction. Dissertation Abstracts International Section A: Humanities \& Social Sciences 2006; 67(4-A):1264.

57. Harrison TC, Stuifbergen AK. Life purpose. Rehabil Nurs. 2006;31(4):149-154.

58. Smith BW, Zautra AJ. Role of purpose in life in recovery from knee surgery. Int J Behav Med. 2004;11(4):197-202.

59. Carroll S. Spirituality and purpose in life in alcoholism recovery. J Stud Alcohol. 1993;54(3):297-301.

60. Chamberlain K, Zika S. Religiosity, life meaning and well-being. J Sci Study Relig. 1988;27(3):411-420.

61. Ellis JB, Smith PC. Spiritual well-being, social desirability and reasons for living. International Journal of Social Psychology. 1991;37(1):57-63.

62. Borrell-Carrio F, Suchman AL, Epstein RM. Biopsychosocial model 25 years later. Ann Fam Med. 2004;2(6):576-582.

63. Wilson IB, Cleary PD. Linking clinical variables with health-related quality of life. JAMA. 1995;273(1):59-65.

64. Burnam MA, Hough RL, Escobar JI, et al. Six-month prevalence of specific psychiatric disorders among Mexican Americans and non-Hispanic Whites in Los Angeles. Arch Gen Psychiatry. 1987;44(8):687-694.

65. Brown JB, Adams ME. Patients as reliable reporters of medical care process. Med Care. 1992;30(5):400-411.

66. Fiscella K. Is lower income associated with greater biopsychosocial morbidity? J Fam Pract. 1999;48(5):372-377. 